Referencia para citar este artículo: Ávila-Navarrete, V. C. (2017). ¿Corresponsabilidad familiar en instituciones de reeducación para adolescentes infractores? Revista Latinoamericana de Ciencias Sociales, Niñez y Juventud, 15 (2), pp. 1191-1206. DOI:10.11600/1692715x.1522712102016

\title{
¿Corresponsabilidad familiar en instituciones de reeducación para adolescentes infractores?*
}

\author{
VIVIANA CARMENZA ÁVILA-NAVARRETE** \\ Profesora Universidad Católica Luis Amigó, Colombia.
}

\begin{abstract}
Artículo recibido en octubre 12 de 2016; artículo aceptado en febrero 27 de 2017 (Eds.)
\end{abstract}
- Resumen (analítico): en el presente artículo expongo los aprendizajes de un plan estratégico para el incremento de la corresponsabilidad familiar en instituciones de reeducación en Colombia. Mediante la metodología de marco lógico propongo estrategias y acciones participativas para que las familias garanticen la atención, el cuidado y la protección de los individuos adolescentes al momento de llevar a cabo un proceso reeducativo integral. El análisis situacional evidenció que el déficit en la corresponsabilidad familiar tiene origen multicausal, con factores extrainstitucionales e intrainstitucionales que influyen y repercuten de manera bidireccional. Concluyo que el trabajo coordinado, articulado y competente entre los diversos profesionales adscritos al Sistema colombiano de Responsabilidad Penal Para Adolescentes, basado en el análisis de relaciones e interacciones, puede proveer elementos necesarios para que la familia reconozca y asuma la corresponsabilidad.

Palabras clave: adolescencia, delincuencia juvenil, conflicto social, corresponsabilidad, formación, reeducación, institución, organización, familia, papel de los padres y madres (Tesauro de Ciencias Sociales de la Unesco).

\section{Family co-responsibility in rehabilitation institutions for adolescent offenders?}

- Abstract (analytical): the present article details the results of the implementation of a strategic plan for increasing family co-responsibility in rehabilitation institutions in Colombia. Using the logical framework methodology, participatory strategies and actions are proposed to ensure that families guarantee assistance, care and protection for adolescents during the period that they are involved in a rehabilitation process. The situational analysis used in the study showed that the deficit in family co-responsibility has a multi-causal origin, with intra-institutional and other factors having a bidirectional influence and impact. The authors conclude that coordinated, articulated and competent work between the different professionals working in the Colombian criminal responsibility system for adolescents, based on the analysis of relationships and interactions, can provide the necessary elements for families to acknowledge and assume their co-responsibility.

Key words: adolescence, juvenile delinquency, social conflict, responsibility, training, reeducation, institution, organization, family, role of parents (Thesaurus of Social Sciences of Unesco).

Este artículo de reporte de caso hace parte de la sistematización del proyecto académico denominado "Plan estratégico para el incremento de la corresponsabilidad familiar en instituciones de reeducación para adolescentes en conflicto con la Ley penal colombiana", del curso gestión de programas para la familia, ofrecido dentro de la Maestría en Asesoría Familiar y Gestión de Programas para la Familia de la Universidad de la Sabana, Chía-Colombia. Fecha de inicio: 24 de febrero de 2014. Fecha de culminación: 25 de noviembre de 2015. Área: ciencias sociales. Subárea: interdisciplinaria.

** Psicóloga, con estudios avanzados en psicología del consumidor, Fundación Universitaria Konrad Lorenz. Diplomada en Estrategias terapéuticas institucionales, Universidad Católica Luis Amigó. Especialista en psicología jurídica, Universidad Santo Tomás. Especialista en pedagogía institucional terapéutica, Universidad Católica Luis Amigó. Magíster en Asesoría Familiar y Gestión De Programas Para La Familia, Universidad de la Sabana. Profesora de la Universidad Católica Luis Amigó, Facultad de Psicología y Ciencias Sociales, Bogotá-Colombia. Orcid: 0000-0002-2701-5769. Correo electrónico: viviana.avilana@amigo.edu.co 


\section{Corresponsabilidade familiar em instituições de reeducação para adolescentes infratores?}

- Resumo (analítico): este artigo apresenta a aprendizagem de um plano estratégico para o aumento da corresponsabilidade famíliar em instituições de reeducação na Colômbia. Por meio da metodologia do marco lógico são propostas estratégias e ações participativas para que as famílias garantam atenção, cuidado e proteção aos adolescentes no momento da realização de um processo de reeducação integral. A análise da situação mostrou que o déficit de co-responsabilidade famíliar tem origem multicausal, com fatores extra e intrainstitucional que influenciam e repercurtem de maneira bidirecional. Conclui-se que o trabalho coordenado, articulado e competente entre os vários profissionais ligados ao sistema colombiano de responsabilidade penal para adolescentes, com base na análise das relações e interações, pode fornecer os elementos necessários para a família a reconhecer e assumir a corresponsabilidade.

Palavras-chave: adolescência, delinquência juvenil, conflito social, corresponsabilidade, educação, responsabilidade social, instituição, organização, família, papel dos pais (Tesauro de Ciências Sociais da Unesco).

-1. Introducción. -2. Reeducación y corresponsabilidad familiar. -3. Fortalecimiento de la corresponsabilidad familiar en el proceso reeducativo: plan estratégico. $\mathbf{- 4}$. Conclusiones. -Lista de referencias.

\section{Introducción}

La ejecución de la Ley 1098 de Infancia y Adolescencia (República de Colombia, 2006) convocó a diversas autoridades y entidades colombianas a fin de proponer acciones y competencias relacionadas con la ejecución de normas y actuaciones para salvaguardar el debido proceso y el restablecimiento de derechos, y avalar la finalidad pedagógica del sistema conforme a la protección integral. Parte de esto, en razón a que los adolescentes y las adolescentes que incursionan en la delincuencia requieren ayuda especializada que desde múltiples instancias provean un tratamiento integral, favorecedor de rehabilitación y de resocialización.

Las instituciones de reeducación, como entidades adscritas al Sistema de Responsabilidad Penal Para Adolescentes ${ }^{1}$ (Instituto Colombiano de Bienestar FamiliarICBF, 2013a), realizan numerosos esfuerzos para optimizar la atención de las gentes

1 El Sistema de Responsabilidad Penal Para Adolescentes -SRPApropuesto en el Art. 139 de la Ley 1098 (2006), se entiende como aquel conjunto de principios, normas, procedimientos, autoridades judiciales especializadas y entes administrativos que rigen o intervienen en la investigación y juzgamiento de delitos o hechos punibles, cometidos por personas que tienen entre catorce (14) y dieciocho (18) años de edad (Instituto Colombiano de Bienestar Familiar-ICBF, 2013a). jóvenes y sus familias. Operan en reciprocidad con el Instituto Colombiano de Bienestar Familiar (Instituto Colombiano de Bienestar Familiar-ICBF, 2015) y con otras entidades del Estado, para dar respuesta ante las sanciones pedagógicas impuestas por los juzgados de infancia y adolescencia, acorde con la misma Ley: amonestación, prestación de servicio comunitario, reglas de conducta, libertad vigilada, vinculación en centro semicerrado, internamiento preventivo, privación de libertad (Instituto Colombiano de Bienestar FamiliarICBF, 2013a).

Las estadísticas presentadas por el Instituto Colombiano de Bienestar Familiar muestran que entre los años 2007-2015, un 38,3\% de los individuos adolescentes colombianos fueron remitidos a una institución de atención especializada, notándose claramente la tendencia al aumento de casos por año. La mayor ocurrencia de infracciones se concentró en las edades de 15 años (22\%), 16 años (29\%) y 17 años $(34 \%)$, siendo las trasgresiones de mayor ocurrencia el hurto y porte de estupefacientes (Instituto Colombiano de Bienestar FamiliarICBF, 2015).

Los datos registrados ponen de manifiesto que, llevar a cabo la labor pedagógica y terapéutica en el contexto reeducativo para la 
resignificación de la noción conflictiva de Ley en sujetos adolescentes infractores, involucra la participación activa de varios actores sociales; implica un arduo compromiso que no solo compete a los equipos interdisciplinarios de las instituciones, sino que se circunscribe a los miembros del grupo familiar.

No obstante, en repetidas ocasiones el proceso reeducativo se ve afectado porque el ciclo de acompañamiento familiar se reduce a gran escala, limitando el curso de acciones restaurativas ante los daños recibidos y causados en la historia de vida de la persona adolescente.

Esta carencia de ayuda o deserción por parte de la familia, produce en el individuo joven un elevado nivel de resistencia para asumir el proceso de atención integral, tornándose renuente a aceptar modificaciones en su comportamiento aunque le sea favorable. Grupalmente aparecen efectos colaterales con comportamientos que perjudican el bienestar del grupo, vulneran la sana convivencia institucional $\mathrm{y}$ trascienden socialmente: desesperanza, matoneo, ansiedad colectiva, brotes de indisciplina, intentos de evasión y amotinamientos.

La realidad expuesta devela peligrosas fallas en el Sistema de Responsabilidad Penal Para Adolescentes en Colombia; una de ellas es la concerniente al déficit en la corresponsabilidad familiar en el proceso reeducativo, siendo notoria la ausencia de cooperación por parte de los miembros de la familia para compartir la responsabilidad de tareas y asumir de manera garante el ejercicio de los derechos.

En razón a lo expuesto emergen varias preguntas: ¿Cómo se determina la corresponsabilidad familiar? ¿De qué manera la corresponsabilidad familiar se ve afectada, cuando los hijos e hijas se encuentran vinculados a una institución de reeducación? ¿Qué razones tiene una familia para no garantizar la protección integral de los hijos e hijas adolescentes dentro del Sistema de Responsabilidad Penal? ¿Se puede incrementar la corresponsabilidad familiar en instituciones de reeducación para adolescentes infractores?

Rev.latinoam.cienc.soc.niñez juv 15 (2): 1191-1206, 2017 http://revistalatinoamericanaumanizales.cinde.org.co

DOI: $10.11600 / 1692715 \times .1522712102016$
Las anteriores cuestiones demandan un amplio sentido de reconocimiento por parte de todos los actores sociales, sobre todo de quienes desarrollan el ejercicio de instruir en la Ley, educar e intervenir pedagógica y terapéuticamente. De ahí que el objetivo principal de este trabajo se centró en proponer un plan estratégico para el incremento de la corresponsabilidad familiar en las instituciones de reeducación para adolescentes infractores en Colombia, favoreciendo la inclusión social y la creación de espacios reflexivos para poder comprender e intervenir sobre la realidad.

\section{Reeducación y Corresponsabilidad Familiar}

Desde el ámbito jurídico, pedagógico y terapéutico, la palabra reeducación se entiende como el acto de volver a enseñar a las personas aquellas conductas socialmente positivas que por diversas circunstancias se han disipado en el tiempo o espacio, y que potencialmente generan inconvenientes en el desarrollo, funcionamiento integral y trascendencia co-existencial.

La efectividad del acto de reeducar depende potencialmente de dos entidades: la institución y la familia. Las instituciones representan la unidad que direcciona los procesos mediante un grupo de individuos expertos con saberes específicos, y las familias representan las personas a quienes se haya dirigido el proceso de tratamiento (Vilar, 2010).

Muchas de las instituciones que sirven programas para adolescentes infractores fundamentan el concepto de reeducación en los postulados de la pedagogía reeducativa ${ }^{2}$, como disciplina de la pedagogía social que ofrece herramientas para mejorar la

2 Esta propuesta surge en el siglo XIX con el Padre Fray Luis Amigó y Ferrer, fundador de la Congregación de Religiosos Terciarios Capuchinos. El apostolado que los ha caracterizado se fundamenta en la pedagogía del amor y la presencia, y los hace pioneros mundiales en el trabajo con población infanto-juvenil en situación irregular, de vulnerabilidad o exclusión social. La primera propuesta de reeducación surgió en el año 1890 en la Escuela de Reforma Santa Rita (Madrid, España). Actualmente la intervención de los Terciarios Capuchinos en las instituciones de reeducación se consolida mediante un proceso de cientificidad desde la metodología psicopedagógica, en la que el sujeto es mirado como un ser transcendente, capaz de transformar su vida (Marín, 2009). 
convivencia social. Otras fuentes corresponden a los postulados de la animación sociocultural, de la pedagogía crítica, de la pedagogía terapéutica y de la pedagogía de la presencia, al posibilitar mecanismos para la transformación, socialización, sanación y restauración (León \& Calderón, 2012; León, Calderón \& Romero, 2010; País, 2012; País \& González, 2014).

De acuerdo con estos postulados, las instituciones de reeducación deben crear ambientes éticos y estéticos, coherentes y organizados, con escenarios benéficos que inviten a construir arraigo y pertenencia; han de incitar a la familia hacia la motivación constructiva y colaborativa en ese nuevo espacio vital, promoviendo el análisis de las interacciones acerca de la situación presentada y el desarrollo integral de quienes allí conviven (Morales, 2004). Deben procurar que las familias promuevan la presencia activa y responsable para adquirir competencia social desde la capacidad para construir afecto, conocimiento y conductas proactivas socialmente.

En este sentido, la corresponsabilidad familiar se convierte en punto esencial para considerar el éxito o fracaso al momento de abordar adolescentes infractores de la Ley penal. Se constituye en herramienta fundamental para las instituciones de reeducación, y su efecto tiene gran impacto cuando las redes familiares se vuelven fuente de apoyo básico y proveen las condiciones adecuadas para que la gente joven pueda concretar sus metas de vida, obrando con rectitud (Instituto Colombiano de Bienestar Familiar-ICBF, 2007, 2013b).

La corresponsabilidad ha sido retomada por Beloff (2001) para referirse a las responsabilidades propias y claramente diferenciadas de todos los actores sociales, para la garantía de los derechos. En Colombia, este concepto se puntualiza en el Art. $10^{\circ}$ del sustento normativo de la Ley de Infancia y Adolescencia de 2006, como la "concurrencia de actores y acciones conducentes a garantizar el ejercicio de los derechos de los niños, las niñas y los adolescentes", siendo la familia, la sociedad y el Estado los encargados de brindar atención, cuidado y protección (República de Colombia, 2006).

Una visión más específica es brindada por Maganto, Bartau y Etxeberría (2004), quienes asumen la corresponsabilidad familiar como los repertorios comportamentales que median en la división, reparto, participación y aprobación en relación con los quehaceres y encargos entre los miembros de una familia. Asimismo, Hoyos (2011) la concibe como un estilo de formación democrática que se extiende más allá de lo realizado por los padres y madres en el núcleo familiar, integrada por principios y elementos que actúan mejorando el desempeño del sistema a través del apoyo constante, el trato dignificante, el amor, el respeto y la aceptación del otro.

Desde estas nociones, la familia se constituye en motor de la corresponsabilidad porque es unidad ecosistémica favorecedora de supervivencia, evolución y cambio (Hernández, 2005), es el ámbito originario para la consolidación y crecimiento de la libertad, $\mathrm{y}$ es escenario educativo para el entramado de relaciones interpersonales que protege y promueve el descubrimiento de la propia identidad a través de metodologías pedagógicas que subyacen a la cotidiana convivencia (De Soria, 2005).

Las interacciones familiares llevan a que las prácticas de corresponsabilidad se sometan a un proceso de construcción donde existen fisuras de percepción entre los sujetos adultos de la familia. Dichas hendiduras van modificándose en tiempo y espacio luego del nacimiento del primer hijo o hija, llevando a que las funciones y los roles entre el padre y la madre posean variadas connotaciones y difieran entre sí (Sánchez, 2012; Villar, Pernas \& Iglesias, 2012).

Esta condición se denomina brecha de corresponsabilidad familiar (Villar et al., 2012) y se halla en función de la estructura familiar, la ideología de género, el empoderamiento del rol, la edad y el sexo de los hijos e hijas. No solo afecta la calidad del tiempo compartido sino la existencia de acuerdos hacia la responsabilidad para el cuidado de 
los descendientes, especialmente durante la infancia y la adolescencia (Ortega, Rodríguez \& Jiménez, 2013).

En virtud de ello, muchos países han diseñado sistemas para la protección de la familia, implementando procedimientos $y$ programas en los que desisten del apelativo de menores referido a los hijos e hijas, al ser un modo descalificante frente a su capacidad de autodeterminación. Estos sistemas pasan a considerar a los niños, niñas y adolescentes como sujetos de derecho, y se convierten en una propuesta reflexiva ante la brecha de corresponsabilidad familiar, sobre todo cuando los individuos adolescentes emprenden un trayecto que los puede llevar al sistema de responsabilidad penal de personas adultas (Beloff, 2000; Cerda \& Cerda, 2006; Fernández, 2015; González-Laurino, 2015; Ortiz, 2009).

En Colombia, la protección integral de la familia se extiende al escenario pedagógico para poder comprender la dinámica y los juegos de interacción en cada uno de los miembros del sistema, acorde con el ciclo evolutivo y el contexto de desenvolvimiento (Hernández, 2005), favoreciendo la educación familiar. Es aquí donde los miembros de la familia, como sujetos de educación, aprenden a convivir con un conjunto de elementos que configuran la vida del otro, conllevando una serie de relaciones interpersonales que median los aprendizajes personales en las dimensiones biopsicosocial, cognitiva, afectiva, ciudadana, ética y moral (De Soria, 2014).

Las instituciones de reeducación y los programas de protección-educación familiar, deben aspirar a regenerar la responsabilidad y el trabajo colaborativo entre todos los miembros de la familia, a partir de la construcción de un modelo participativo cimentado en la solidaridad intergeneracional, la autonomía, la satisfacción afectiva y la regulación emocional (Peña, Menéndez \& Torío, 2010). Alcanzar los resultados esperados obliga a trabajar sobre tres dimensiones básicas de la corresponsabilidad familiar, en las que cada actor social debe ser y hacerse protagonista (Maganto et al., 2004):
1) La coherente $y$ equitativa distribución de tareas para cada uno de los miembros de la familia, teniendo en cuenta sus necesidades, características y posibilidades, evitando la sobrecarga de roles para que las tareas sean relativas a la administración del hogar. Esta dimensión implica un esfuerzo integral de todos los miembros de la familia que aportan en la formación de los adolescentes y las adolescentes, y una excelente gestión de los recursos disponibles.

2) La acción coordinada $y$ armónica entre los miembros de la familia para dar cumplimiento a las tareas $\mathrm{y}$ llevar a cabo lo concertado, propendiendo siempre por el respaldo conjunto. Esta dimensión favorece el reconocimiento de la capacidad de cada uno de los miembros de la familia para crear, sortear situaciones y tomar decisiones, aun cuando existan debates y cuestionamientos por parte de los otros.

3) El apoyo emocional $y$ motivacional a partir de la connotación positiva frente a la tarea efectuada, la responsabilidad compartida, el bienestar del sistema familiar y la organización como unidad integralizante. Esta dimensión ayuda en la construcción del si-mismo de cada uno de los miembros de la familia, en la trascendencia personal y en el fortalecimiento de las relaciones de confianza.

Al respecto, León et al. (2010) propone que la política de protección, educación y corresponsabilidadenla familiadebe aventurarse al adecuado cumplimiento de las funciones vitales para optimizar la calidad de vida familiar y prevenir la aparición de los distintos problemas sociales. Este ejercicio apela a equipos de profesionales que puedan interactuar interdisciplinaria y multidisciplinariamente frente a la atención integral, manteniendo un claro enfoque en el diseño, intervención, implementación, supervisión y evaluación de programas para la familia (Jiménez, 2003; Hernández, 2005). 


\section{Fortalecimiento de la corresponsabilidad familiar en el proceso reeducativo: plan estratégico}

\subsection{Generalidades}

El plan para mejorar la corresponsabilidad familiar emerge de un acumulado de experiencias a la luz del trabajo profesional en la Congregación de Religiosos Terciarios Capuchinos, con los usuarios y usuarias del Sistema de Responsabilidad Penal Para Adolescentes que se encuentran vinculados en una institución de reeducación de la ciudad de Bogotá, Colombia.

Las diversas situaciones y acontecimientos vivenciados durante 15 años de labor, motivaron el estudio y análisis de la corresponsabilidad familiar. Por ello, desde el inicio el plan estratégico se proyectó como una composición co-constructiva para la consecución de prácticas formativas y terapéuticas, a propósito de las exigencias institucionales que subyacen a las transformaciones sociales y al compromiso de quienes creemos en la reeducación.

El ejercicio se fundamentó en una aproximación retrospectiva de vivencias, rutinas, rituales, prácticas y procesos sociales, examinando el sentido de la corresponsabilidad familiar: ideas, conceptos, interpretaciones y proposiciones. Se hizo especial énfasis en la profundización de saberes, costumbres y juicios de la comunidad, centrándose en las vicisitudes de la contemporaneidad.

En el año 2014 se concretó la formulación del diagnóstico situacional como soporte argumentativo de la experiencia, y en el año 2015 se instó al ejercicio de planeación estratégica, rescatando prácticas significativas desde la acción participante como medio de conocimiento. Se esbozaron acciones para contrarrestar el déficit en la corresponsabilidad familiar, sin desconocer que existen otras que no se contemplan desde lo escrito y que al momento de hacer lo pedagógico terapéutico son funcionales a partir del conocimiento, el arte y la convicción de una labor.
Todo el proceso de cualificación se encuadró desde el paradigma sistémico, divisando aspectos en proporción a la justicia social y restaurativa. Este fundamento permitió una mejor comprensión alrededor de los patrones de comportamiento y formación en la familia, como actor dinámico dentro de los diversos escenarios de convivencia.

\subsection{Antecedentes}

El tema de corresponsabilidad familiar ha sido poco estudiado a nivel nacional e internacional. La revisión detallada de la literatura presenta escasa información sobre experiencias similares. Para este reporte de caso consideré los siguientes:

- Programa de corresponsabilidad familiar Cofami para fomentar la cooperación y la responsabilidad de los hijos en el trabajo y la vida familiar (Maganto et al., 2004). Ofrece un plan de formación para madres y padres mediante estrategias educativas, siguiendo la estructura de formación en grupo a nivel de educación axiológica y responsabilidad familiar compartida.

- Programa del Ministerio de Educación Nacional ¿cómo participar en los procesos educativos de la Escuela? (Ministerio de Educación Nacional-MEN, 2007). Permite reflexionar colectivamente sobre la forma como los padres y madres o responsables de los niños y niñas pueden participar en el proceso educativo, mediante unos temas centrales sobre los que se enuncian interrogantes para ser analizados y concertar relaciones de participación social.

- Programade educación parental "construir lo cotidiano" (López, Calvo, Menéndez, García \& Martín, 2010). Pretende revisar las concepciones que tienen los padres y madres acerca de la educación otorgada a los hijos e hijas, en perspectiva de los vínculos, de las tareas asignadas y de las responsabilidades, fomentando hábitos de igualdad, solidaridad y responsabilidad compartida. 
- Proyecto corresponsabilidad del maestro de hoy frente a los paradigmas emergentes, para la reconfiguración del tejido social (Aguirre-García \& Muñoz-Orozco, 2012). Brinda una serie de prácticas fundamentadas en el uso de metáforas para la búsqueda e identificación del encargo formativo, con la colaboración de otros agentes sociales.

- Proyecto de corresponsabilidad desde la educación personalizada en el Inem José Celestino Mutis (Fitzgeral-Gómez, 2013). Establece una iniciativa de diálogo dinámico sobre la importancia social de la corresponsabilidad en diversos contextos y escenarios, involucrando a todos los entes institucionales, especialmente a los padres y madres de familia.

- Modelo operativo de gestión de redes sociales para el sistema penal adolescente de Chile (Andrade-Gyllen, García-Ojeda \& Zambrano-Constanzo, 2016). Propone la ejecución de pautas beneficiosas en tiempo y espacio, alrededor de la responsabilidad social y el trabajo en red, a fin de favorecer la sensibilización, la cooperación y la integración social en los sujetos adolescentes infractores.

\subsection{Contexto}

El lugar donde se expresó el problema relacionado con el déficit en la corresponsabilidad familiar y en el que se posibilitó el estudio de caso, fue el Centro Educativo Amigoniano (CEA) ubicado en la ciudad de Bogotá, Colombia, el cual es dirigido por la Congregación de Terciarios Capuchinos.

Es una institución de reeducación sin ánimo de lucro, que ofrece un servicio de atención integral a los individuos adolescentes y jóvenes infractores de la ley que han sido remitidos por autoridades competentes, para que junto con sus familias dinamicen acciones participativas y transformadoras desde la perspectiva de la pedagogía Amigoniana y la inclusión social.

Se hace relevante mencionar que el plan estratégico para el incremento de la corresponsabilidad familiar tiene amplia cobertura y alcance, y se puede extender a otros contextos de naturaleza educativa, preventiva o protectiva. Ofrece una contribución con visión estratégica no solo para la implementación de toda la gestión pedagógica terapéutica, sino para la incentivación de reflexiones por parte de quienes tienen el reto de llevar a cabo las prácticas institucionales, pues hacer pedagogía reeducativa y educación familiar implica una labor de reingeniería y empoderamiento profesional-vocacional.

\subsection{Participantes}

En el ejercicio participaron diversos actores del Sistema de Responsabilidad Penal Para Adolescentes (Instituto Colombiano de Bienestar Familiar-ICBF, 2013b), que influyen en los procesos de corresponsabilidad familiar, entre ellos:

- Actores evidentes: adolescentes vinculados al programa reeducativo, su familia nuclear, lineal y/o extensa, y los equipos interdisciplinarios pertenecientes a la institución de reeducación.

- Actores potenciales: integrantes de la comunidad barrial, miembros del Instituto Colombiano de Bienestar Familiar, Policía de Infancia y Adolescencia, Jueces de Infancia y Adolescencia, entre otras Entidades del Estado.

La metodología que utilicé no involucró riesgos para personas ni otros sujetos. Le otorgué especial importancia a suministrar información acerca de los fines académicos e investigativos, favoreciendo la participación voluntaria, el respeto a la dignidad, y la libertad.

A fin de evitar posibles señalamientos, ofrecí garantía de anonimato a los sujetos participantes, salvaguardando su privacidad.

\subsection{Descripción}

Consolidé el plan estratégico partiendo de la metodología de marco lógico hasta llegar al proceso de sistematización de la experiencia: 
Momento 1. Descripción de la plataforma de gestión. Inicié el procedimiento con un reconocimiento del direccionamiento estratégico y de la aprehensión de los principios corporativos; indagué sobre el interés de participación institucional.

Momento 2. Diagnóstico estratégico $y$ apreciación situacional. Llevé a cabo la obtención de información mediante técnicas e instrumentos específicos, entre los cuales prevalecieron:

- Mesa técnica: reunión previa con actores sociales e integrantes de la comunidad, a fin de conocer las características del caso y entender la realidad (Muñoz \& Muñoz, 2001).

- Taller reflexivo: herramienta educativa, crítica, dialógica, socializadora y concientizadora, que posibilita la deliberación temática y el desenvolvimiento en equipo a través de una postura integradora teórico-práctica (Torres \& Navarro, 2014).

- Grupo focal: encuentro con modalidad de entrevista grupal donde procuré que los participantes discutieran y elaboraran una temática a partir de la experiencia personal (Hamui-Sutton \& Varela-Ruiz, 2013).

- Evaluación de aprendizaje fundamentado en competencias colaborativas: valoración didáctica a modo de proyectos, que desarrolla el interés por el aprendizaje y la producción de conocimientos más allá de lo teórico (Maldonado, 2008).

- Test de la familia: prueba de evaluación proyectiva en la que a partir del dibujo de la familia se enfatizó en detalles de interés para el estudio del sistema familiar y los diferentes personajes que transversalizan el contexto (Lluis-Font, 2012).

- Registro de entrevista: cumplió la función de elemento guía para instaurar la conversación con diversos actores sociales, mediante una serie de preguntas sobre la corresponsabilidad familiar.

- Registro de asistencia: formato en el que las familias y adolescentes consignaron la participación en distintas actividades.

Involucré diversas fuentes de información con participantes vinculados en el proceso pedagógico y terapéutico integral. Particularmente forjé una exploración de la realidad social que subyace al problema de la no corresponsabilidad familiar, para poder establecer un punto de referencia y entender el contexto de manera amplia.

Interpreté los discursos a fin de consolidar una lectura social y construir el árbol de problemas y de objetivos (Camacho, Cámara, Cascante \& Sainz, 2001).

Posteriormente llevé a cabo el análisis estratégico situacional, mediante una matriz Foda con el respectivo inventario de fortalezas, oportunidades, debilidades y amenazas (Carvajal, 2010; Comisión Económica Para América Latina y el Caribe-Cepal, 1998); paralelamente elaboré los diseños de vectores de descripción del problema (VDP), los cuales plasmé en múltiples flujogramas de estudio, tal como se evidencia en el ejemplo de la figura 1.

Con estas herramientas generé una representación de aquellos aspectos deseables y no deseables asociados al problema; es decir, describí la situación desde diversos escenarios, numerando hechos concretos que verifican su existencia (Huertas, 1996) y que pueden ser abordados mediante proyectos y actividades. 
Figura 1. Flujograma VDP.

\section{Problema: Inasistencia por no tener quien cuide a los otros hijos \\ Actor: Familia de un adolescente vinculado en el programa reeducativo}

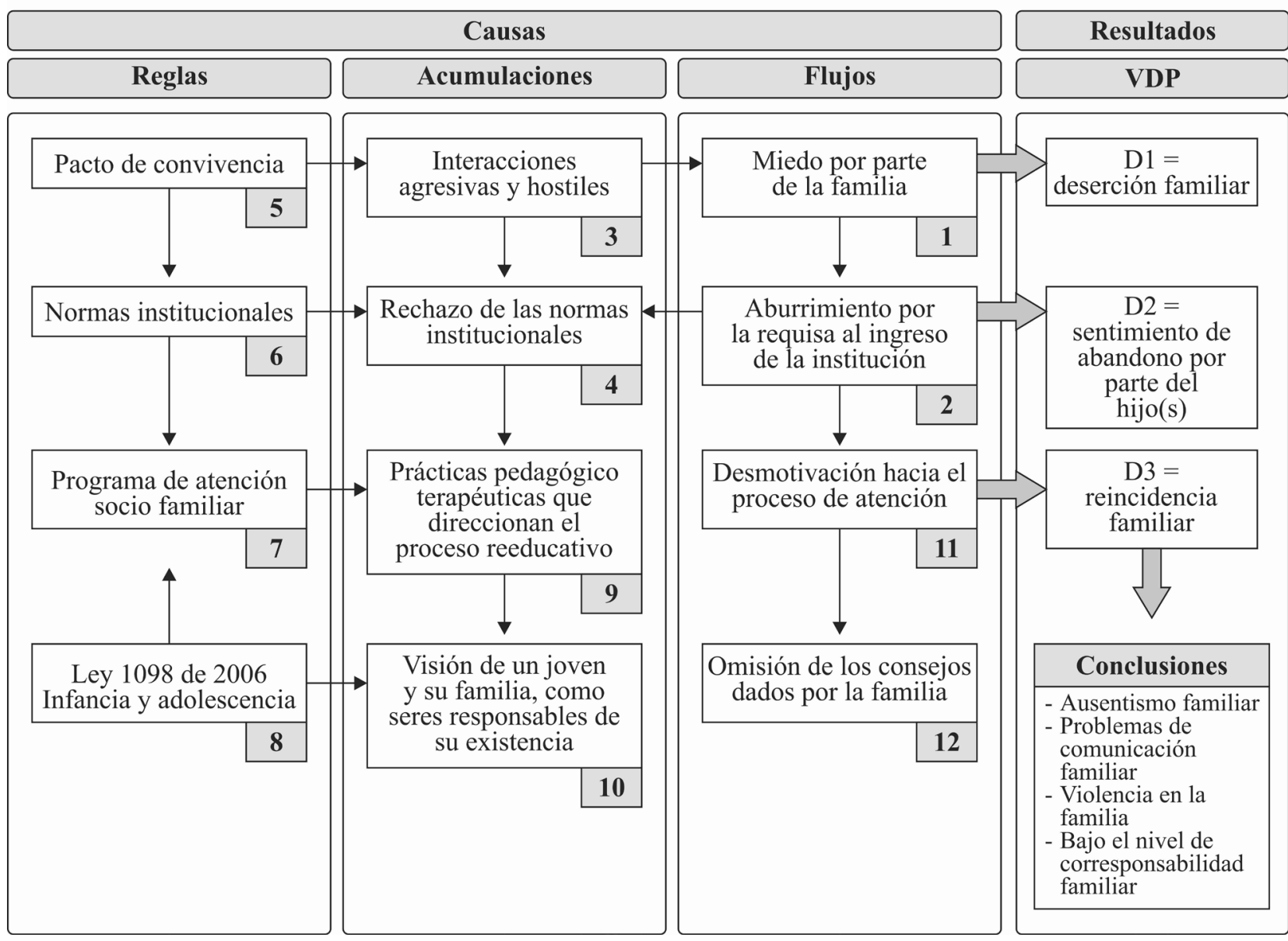

Fuente: elaboración propia.

La lectura de la realidad indicó que existen factores extrainstitucionales e intrainstitucionales que influyen y repercuten de manera bidireccional en el déficit de corresponsabilidad familiar, al interior de la institución de reeducación para adolescentes infractores.

Dentro de la génesis extrainstitucional se destacaron factores relacionados con precarios recursos socioeconómicos en la familia, cansancio y estrés social, condiciones de exclusión, rechazo de la comunidad, habitabilidad y entorno residencial riesgoso, estructura y ambiente familiar disfuncional, trayectoria familiar oposicionista y/o delictiva, conflictos en la comunicación, violencia intrafamiliar, percepción de desigualdad social, indiferencia social por parte de algunas entidades del Estado, subordinación de la familia y de los miembros adolescentes ante algunos actores potenciales que hacen parte del Sistema de Responsabilidad Penal, y percepción de desarticulación entre la realidad social y las acciones expresadas como mandato de Ley.

A nivel intrainstitucional, se acentuaron factores alrededor de la inestabilidad en la organización y dinámica institucional, predominio en juegos de poder jerárquico por parte de jefaturas inmediatas, poca experticia y competencia profesional por parte de algunos miembros de equipos interdisciplinarios, activismo institucional que no permite alternar iniciativas para el uso del tiempo libre, presencia de problemas psicopatológicos en 
varios individuos adolescentes, intransigencia de la familia ante las indicaciones disciplinarias o de autoridad, desorientación jurídica por parte de la comunidad adolescente, tiempos largos de sanción y de permanencia en la institución, además del consumo de sustancias psicoactivas.

Momento 3. Estudio de los actores. Los actores evidentes y potenciales del Sistema de Responsabilidad Penal Para Adolescentes fueron agentes principales en la participación, control e influencia sobre las numerosas situaciones que se concibieron alrededor de la corresponsabilidad familiar. Por esto desplegué un análisis frente a cada uno de ellos, considerando la demanda que tenían a nivel social, sus intereses, el impacto y actitud ante el proyecto, además de la jerarquía de poder (Cano, 2004).

Momento 4. Gestión de riesgos: Adelanté un reconocimiento de los factores externos que, al ser identificados a tiempo, permitían instaurar gestiones preventivas y correctivas para alcanzar los objetivos esperados (Serna, 2000). En este análisis tuve en cuenta los procesos institucionales, las causas y efectos del riesgo, su propiedad y la forma de mitigación.

Momento 5. Matriz de planificación: Con la información obtenida hasta el momento diseñé una matriz de marco lógico (Ortegón, Pacheco \& Prieto, 2005) con la formulación de los fines del proyecto, los propósitos, las actividades y los resultados esperados. Crucé estos lineamientos con el planteamiento de la lógica de la intervención, los indicadores de gestión, los supuestos y las fuentes de verificación.

Momento 6. Plan de acción y planeación de actividades especificas. En concordancia con la matriz de planificación, hice el levantamiento de un plan de acción con la estructuración de una miscelánea de actividades de intervención participativa, en función de las siguientes estrategias:

- Implementación del Modelo Solidario de Inclusión Social ${ }^{3}$

3 Los lineamientos del Modelo Solidario "constituyen el marco paradigmático, conceptual, metodológico y normativo que orienta el trabajo con las familias, para cumplir con la misión institucional con una perspectiva ecosistémica y de complejidad" (Instituto Colombiano de Bienestar Familiar-ICBF, 2007, p. 6).
- Consolidación del cuerpo profesional para la realización de la misión y objetivos estratégicos institucionales

- Procesos decapacitación-formación para todos los colaboradores de la institución en el Sistema de Responsabilidad Penal Para Adolescentes

- Construcción de redes sociales interinstitucionales

- Utilización de redes de comunicación y conversación interpersonal

- Intervenciones individuales y grupales

- Actualización del saber sobre las transformaciones de la familia

- Establecimiento de redes vinculares usuarios-familias-institución

Momento 7. Indicadores de gestión. Este momento me facilitó la exploración y el estudio en relación con los fines y propósitos trazados, el cumplimiento de las políticas de la institución y el análisis objetivo acerca del problema sobre la disminución de la corresponsabilidad familiar (Armijo \& Pública, 2009).

Momento 8. Análisis de la gestión integral. Incluyó el estudio de la pertinencia, la eficacia, la eficiencia, el impacto y la viabilidad del proyecto en mención.

Como aporte final incorporé una serie de talleres reflexivos como guía en el proceso de educación familiar, a partir de la interacción y búsqueda de nuevos significados ante la realidad del sujeto adolescente y de los demás miembros del sistema.

\subsection{Análisis de la experiencia técnica y metodológica}

La implementación y puesta en marcha de cada momento del plan estratégico, se convirtió en un ejercicio de construcción participativa para edificar conocimiento colectivo mediante redes de comunicación y conversación interpersonal. En sí mismo y a través de los vectores de descripción del problema, el proyecto activó procesos de comunicación entre los individuos participantes, y puso en evidencia diferentes tipos de saberes para poder llegar a una representación de familia al interior de la institución. 
El desarrollo de acciones relacionadas con las entrevistas a profundidad, grupos focales y talleres reflexivos, fue esencial para abrir caminos desde el trabajo colaborativo, facilitando que las familias y sus miembros adolescentes lograran consolidar nuevas lecturas y visiones frente a la corresponsabilidad familiar, en circunstancias de infracción a la Ley. En este sentido, las actividades planeadas fueron convenientes para facilitar la re-significación de la noción preliminar de corresponsabilidad, como forma de acompañamiento asistencialista, consiguiendo que se asumiera desde la idea de responsabilidad compartida con efectos sobre el otro, sobre el mundo social y sobre el entorno próximo y lejano.

Una mínima parte de las familias participantes no logró conexión con la propuesta e hizo manifiesta la desmotivación y la deserción. Esta situación sugiere que el proyecto deba extender las acciones estratégicas desde la dimensión de intervención psicosocial, e incluya en la descripción un nuevo momento denominado estudio de deserción para las familias, el cual ayuda a prever circunstancias latentes y/o emergentes.

En relación con las actividades planeadas para los demás actores sociales, fue eficaz la vinculación de los equipos interdisciplinarios afines al Centro de Reeducación, en tanto aportaron memoria histórica frente a los procesos de corresponsabilidad familiar en la institución. No obstante, algunas acciones insertas en el trabajo de campo para obtener consentimiento informado y datos estadísticos sobre corresponsabilidad en el Sistema de Responsabilidad Penal para Adolescentes, se vieron obstruidas por la demanda en la tramitología desde varias entidades del Estado. El hacer práctico indica que no fue funcional realizar entrevistas a las autoridades competentes; éstas se pueden modificar y direccionar hacia otros actores del sistema, como los directores de los Centros de Reeducación, quienes construyen red social con dichas entidades.

\section{Conclusiones}

Tras la formulación y análisis del plan estratégico, concluyo lo siguiente:

1. En el contexto reeducativo colombiano para sujetos adolescentes infractores de la Ley penal, la corresponsabilidad familiar se entiende como el conjunto de actuaciones parentales que, siendo funcionales $y$ saludables, proporcionan herramientas y competencias para establecer vínculos afectivos y atender las necesidades de los adolescentes, facilitando el proceso reparativo y restaurativo.

Dicha corresponsabilidad se incrementa a medida que la familia establece procesos más humanizados y generadores de cambio, fundamentados en la comprensión de las interacciones hilvanadas en la cotidianidad. Contrario a ello, la corresponsabilidad se desvanece cuando los miembros de la familia se pierden como acompañantes y gestores proactivos, cuando los padres y/o madres no logran reflexionar acerca de las interacciones en su historia de vida, y cuando la sociedad en general asume la sanción o pena judicial como única alternativa ante los problemas de la contemporaneidad.

En las instituciones de reeducación, la corresponsabilidad familiar se convierte en componente pedagógico y terapéutico esencial para la formación de la responsabilidad social, de la adaptación o del ajuste psicológico de los individuos adolescentes y demás miembros de la familia, aspecto que confirma lo propuesto por Maganto et al. (2004) y López et al. (2010) a través del programa Cofami y de educación parental "construir lo cotidiano".

2. La complejidad de los sujetos de la acción reeducativa hace imperante la necesidad de contar con profesionales con perfil y formación especializada, capaces de responder jurídica, pedagógica y psicosocialmente ante los retos que en la actualidad plantean los individuos adolescentes infractores y sus familias. El nivel de competencia profesional permite el acto de identificar las necesidades, problemas y motivaciones de los 
miembros del sistema, beneficiando el reconocimiento de cada quien como persona en continuo aprendizaje.

Todas las personas profesionales vinculadas al Sistema de Responsabilidad Penal Para Adolescentes (Instituto Colombiano de Bienestar Familiar-ICBF, 2013b) están llamadas a ahondar en la identificación de las características diferenciales de cada familia y sus escenarios de interacción. La gestión en mención se desarrolla a partir de relaciones afectuosas, cargadas de compromiso y amor exigente, de manera tal que provea elementos suficientes para que la familia asuma el cuidado y protección integral de los individuos adolescentes, apropiándolos como sujetos de derechos y deberes protagonistas de la responsabilidad social (González-Laurino, 2015).

3. Concebir adecuadas estrategias para incrementar la corresponsabilidad familiar obliga a todos los actores $\mathrm{y}$ profesionales a asumirse y a asumir al otro como ser social. Esta tarea implica el desarrollo de habilidades para entender la complejidad de la esencia humana y de las interacciones colectivas, naturaleza a través de la cual es posible descubrir a la familia que se encuentra oculta en la historia para favorecer su nueva realidad.

Los señalamientos expuestos respaldan las premisas del Ministerio de Educación Nacional en el programa de participación de procesos educativos en la escuela, al insistir en que todas las instituciones pedagógicas deben convertirse en el espacio natural donde la familia encuentra nuevas o mejores oportunidades para reflexionar y empoderarse del ejercicio de roles en la formación de los hijos e hijas (Ministerio de Educación Nacional-MEN, 2007), en tanto se vuelven corresponsables en la construcción de valores junto con el resto de la comunidad educativa, y los entes judiciales y administrativos.

4. Edificar un proceso reeducativo dialógico, colaborativo, interactivo y constructor de nuevas posibilidades con familias corresponsables, requiere de la formación de profesionales para que favorezcan las mediaciones lingüísticas materializadas en dispositivos de lenguaje. Así las cosas, todos los actores adscritos al Sistema de Responsabilidad Penal Para Adolescentes necesitan fortalecer procesos co-constructivos, articulados y complementarios (Beloff, 2001) que partan del complejo momento de intercambio en el que cada actor social acompaña la responsabilidad por el contexto que surge durante el espacio de intercambio con el sujeto adolescente y su familia.

Tal como lo sugiere Aguirre-García y Muñoz-Orozco (2012) en el proyecto sobre corresponsabilidad de los maestros y maestras de hoy, la labor de todos los actores sociales vinculados al proceso reeducativo de los individuos adolescentes infractores debe trascender los límites de la institución. Este mecanismo propende por la transformación de los roles para llegar con mayor eficiencia y eficacia a la población adolescente en conflicto con la Ley; a su vez, motiva hacia la búsqueda de respuestas ante la emergencia de un nuevo paradigma asociado a la nueva comprensión de lo humano, lo jurídico y lo sociopolítico (Aguirre-García \& Muñoz-Orozco, 2012).

5. De cualquier modo, se hace necesario reiterar que las instituciones de reeducación se convierten en organizaciones que tienen un efecto significativo en los procesos de transformación y trascendencia para las personas adolescentes y sus familias; por ello resulta indispensable conducir acciones mancomunadas desde la educación familiar, que permitan a las familias y a la comunidad en general tomar conciencia de las bondades frente a los servicios brindados en las instituciones de reeducación, más allá del cumplimiento de una sanción o medida jurídica asignada por la comisión de un delito.

Como se describe en el modelo operativo de gestión de redes sociales (Andrade-Gyllen et al., 2016), una relación basada en canales de interacción horizontal que propicien la 
comunicación y el respeto por la diferencia entre los actores sociales que trabajan con adolescentes infractores y sus familias, es fundamental en el proceso de construcción social dentro del proceso reeducativo y la activación o regulación ante los complejos fenómenos sociales.

Estos planteamientos corroboran las manifestaciones de Fitzgeral-Gómez (2013) en el proyecto de corresponsabilidad desde la educación personalizada, acerca de la necesidad de articular redes, no solo porque el acto de reeducar se concreta en la interacción involucrando diferentes estamentos, elementos pedagógicos, conceptuales y metodológicos, sino porque el Sistema de Responsabilidad Penal Para Adolescentes (Beloff, 2001) no puede separarse de la educación familiar para poder enfrentar el mundo simbólico que está marcando y direccionado la actividad humana.

Finalmente, y como recomendación, aconsejo avanzar en el estudio de prácticas pedagógicas y terapéuticas dirigidas a atenuar los imaginarios aversivos que se tienen sobre las instituciones de reeducación, y con esto, frenar el impacto negativo acerca de la vinculación de un individuo adolescente en el Sistema de Responsabilidad Penal. De igual forma invito a continuar en la profundización y comprensión del tema sobre la corresponsabilidad familiar, el cual ha sido poco estudiado en Colombia y Latinoamérica.

\section{Lista de referencias}

Aguirre-García, S. E. \& Muñoz-Orozco, L. M. (2012). La corresponsabilidad del maestro de hoy frente a los paradigmas emergentes, para la reconfiguración del tejido social. Tesis para optar al título en maestría en educación, Facultad de Educación, Universidad Católica de Manizales, Manizales, Colombia.

Andrade-Gyllen, C.; García-Ojeda, M. \& Zambrano-Constanzo, A. (2016). Modelo Operativo de gestión de redes sociales para el sistema penal adolescente, Chile. Revista Latinoamericana de Ciencias Sociales, Niñez y Juventud, 14 (1), pp. 247-260. Doi :10.11600/1692715x.14116101214.
Armijo, M. \& Pública, G. (2009). Manual de planificación estratégica e indicadores de desempeño en el sector público (versión preliminar). Santiago de Chile: Ilpes, Cepal. Recuperado de: http://www. cepal.org/ilpes/noticias/paginas/3/38453/ manual_planificacion_estrategica.pdf

Beloff, M. (2000). Los sistemas de responsabilidad penal juvenil en América Latina. En M. García; E. Méndez \& M. Beloff (eds.) Infancia, ley y democracia en América Latina. Análisis crítico del panorama legislativo en el marco de la Convención Internacional sobre los Derechos del Niño (1990-1998), (pp. 23-43). Santiago de Chile: Fondo de las Naciones Unidas para la Infancia. Recuperado de: http://www.cesc.uchile.cl/

Beloff, M. (2001). Algunas confusiones en torno a las consecuencias jurídicas de la conducta transgresora de la ley penal en los nuevos sistemas de justicia juvenil latinoamericanos. En E. GarcíaMéndez (eds.) La dimensión política de la responsabilidad penal de los adolescentes en américa latina: notas para la construcción de una modesta utopía, (pp. 85-102). Buenos Aires: Fondo de las Naciones Unidas para la Infancia.

Camacho, H.; Cámara, L.; Cascante, R. \& Sainz, H. (2001). El Enfoque del marco lógico: 10 casos prácticos. Cuaderno para la identificación y diseño de proyectos de desarrollo. Madrid: Fundación Cideal.

Cano, O. L. (2004). Metodología para la concertación de los actores en la gestión de proyectos de desarrollo. Bogotá, D. C.: Escuela Superior de Administración Pública, Facultad de investigaciones.

Carvajal, A. (2010). Planeación participativa: Diagnóstico, plan de desarrollo $y$ evaluación de proyectos. Santiago de Cali: Facultad de humanidades, Universidad del Valle.

Cerda, M. \& Cerda, R. (2006). Sistema de responsabilidad penal para adolescentes. Santiago de Chile: Librotecnia.

Comisión Económica Para América Latina y el Caribe-Cepal(1998). Gestión deprogramas 
sociales en América Latina, serie politicas sociales 25. Santiago de Chile: Naciones Unidas, Cepal. Recuperado de:

http://repositorio.cepal.org/bitstream/ handle/11362/6255/S9800061_ es.pdf?sequence $=1$

De Soria, A. (2005). La familia como ámbito educativo. Madrid: Rialp.

De Soria, A. (2014). Algunas ideas básicas sobre la dimensión educativa de la vida familiar. Bogotá, D. C.: Universidad de la Sabana. Recuperado de:

https://issuu.com/carolinadb/docs/ algunas_ideas_basicas

Fernández, L. (2015). Análisis de las estrategias de reintegración del adolescente infractor en el Centro de Atención Especializada el Redentor de Bogotá. Tesis para optar al título de abogada, Facultad de Derecho, Centro de Investigaciones Socio-Jurídicas, Universidad Libre, Bogotá, D. C., Colombia.

Fitzgeral-Gómez, M. (2013). La corresponsabilidad desde la mirada de la educación personalizada en el Inem José Celestino Mutis. Tesis para optar al título de especialista en educación personalizada, Universidad Católica de Manizales, Manizales, Colombia.

González-Laurino, C. (2015). La infracción adolescente a la ley penal en el centro del debate sobre seguridad pública en Uruguay. Revista Latinoamericana de Ciencias Sociales, Niñez y Juventud, 13 (2), pp. 951-962. Doi:10.11600/169271 $5 x .13228201014$.

Hamui-Sutton, A. \& Varela-Ruiz, M. (2013). La técnica de grupos focales. Investigación en Educación Médica, 2 (5), pp. 55-60.

Hernández, Á. (2005). La familia como unidad de supervivencia, de sentido y de cambio en las intervenciones psicosociales: intenciones y realidades. Revista Latinoamericana de Ciencias Sociales, Niñez y juventud, 3 (1), pp. 57-71.

Hoyos, C. (2011). La Corresponsabilidad familiar: rupturas históricas y prospectiva en Medellín. Medellín: Unaula.
Huertas, F. (1996). Elmétodo PES: planificación estratégica situacional. Entrevista a Carlos Matus. La Paz: Fondo Editorial Altadir.

Instituto Colombiano de Bienestar FamiliarICBF (2007). Lineamientos técnicos para la inclusión y atención de familias. Bogotá, D. C.: Unidad de prensa e información pública de la OIM. Recuperado de:

http://www.icbf.gov.co/portal/page/portal/ Descargas1/nuevoLineamientos TInclusinAtencionFamilias.pdf

Instituto Colombiano de Bienestar FamiliarICBF (2013a). El ABC del Sistema de Responsabilidad Penal para Adolescentes 1. Guía para su comprensión. Bogotá, D. C.: Unidad de prensa e información pública de la OIM. Recuperado de:

http://www.icbf.gov.co/portal/page/portal/ PortalICBF

Instituto Colombiano de Bienestar FamiliarICBF (2013b). La Familia en el Sistema de Responsabilidad Penal para Adolescentes 3. Guía para su comprensión. Bogotá, D. C.: Unidad de prensa e información pública de la OIM. Recuperado de:

http://www.icbf.gov.co/portal/page/portal/ PortalICBF/Especiales/prueba/Bienestar/ SRPA/p3_SRPA.pdf

Instituto Colombiano de Bienestar FamiliarICBF (2015). Estadística del Sistema de Responsabilidad Penal para Adolescentes. Bogotá, D. C.: Subdirección de Responsabilidad penal. Recuperado de: http://www.icbf.gov.co/portal/page/portal/ IntranetICBF/organigrama/Proteccion/ CifrasSRPA/2015/BOGOTA_11.pdf

Jiménez, F. (2003). El concepto de familia en las instituciones: una mirada de la institución familiar en la Constitución colombiana $e$ interpretación jurisprudencial. Foro sobre Derecho de Familia, Democracia y Derechos Humanos, Secretaría de Gobierno, Personería Distrital, Universidad de los Andes, Bogotá, D. C., Colombia.

León, O. \& Calderón, J. (2012). Aula para educadores. Reflexiones Amigonianas. Bogotá, D. C.: Congregación de Religiosos Terciarios Capuchinos de Nuestra Señora de los Dolores, Provincia San José. Recuperado de: 
¿CORRESPONSABILIDAD FAMILIAR EN INSTITUCIONES DE REEDUCACIÓN PARA ADOLESCENTES INFRACTORES?

http://www.terciarioscapuchinossanjose. org/publicaciones.php

León, O.; Calderón, J. \& Romero, S. (2010). Aproximación a los referentes teóricos y conceptuales que fundamentan la propuesta pedagógica amigoniana. Bogotá, D. C.: Congregación de Religiosos Terciarios Capuchinos de Nuestra Señora de los Dolores, Provincia San José. Recuperadode: http://www.terciarioscapuchinossanjose. org/publicaciones.php

López, S. T.; Calvo, J. V. P.; Menéndez, M. D. C. R.; García, C. M. F. \& Martín, S. M. (2010). Hacia la corresponsabilidad familiar: construir lo cotidiano. Un programa de educación parental. Educatio Siglo XXI, 28 (1), pp. 85-108.

Lluis-Font, J. M. (2012). Test de la familia: cuantificación y análisis de variables socioculturales y de estructura familiar para profesionales de Psicología, Psicopedagogía y Psiquiatría. Barcelona: Davinci. Recuperado de: http://www. infocoponline.es/

Maganto, J. M.; Bartau, I. \& Etxeberría, J. (2004). La corresponsabilidad familiar (Cofami): cómo fomentar la cooperación y la responsabilidad de los hijos. Infancia y Aprendizaje, 27 (4), pp. 417-423.

Maldonado, M. (2008). Aprendizaje basado en proyectos colaborativos. Una experiencia en educación superior. Revista de Educación, 14 (28), pp. 158-180. Recuperado de: http://www. memoriasciebc.com/

Marín,O.D.B.(2009).Brevehistorialapedagogía reeducativa y su relación con la psicología, a la luz del Amigonianismo. Revista Poiésis, 9 (17), pp. 1-10. Doi: http://dx.doi. org/10.21501/16920945.198. Recuperado de:

http://www.funlam.edu.co/revistas/index. $\mathrm{php} / \mathrm{poiesis/article/view/198}$

Ministerio de Educación Nacional-MEN (2007). Cartilla para padres de familia, ¿cómo participar en los procesos educativos de la Escuela? Guía No 26. Bogotá, D. C.: Revolución Educativa Colombia Aprende, Sanmartín Obregón y Cía, ltda. Recuperado de: http://www.mineducacion.gov.co/1759/ articles-120646 archivo pdf.pdf

Morales, S. (2004). Una década de oportunidades: jun tiempo invaluable de aprendizajes! Revista de Derecho: División de Ciencias Jurídicas de la Universidad del Norte, 22 (22), pp. 317-321.

Muñoz, P. \& Muñoz, I. (2001). Intervención de la familia. Estudios de casos. En G. PérezSerrano (coord.) Modelos de investigación cualitativa en educación socialy animación sociocultural-Aplicaciones prácticas. Madrid: Narcea.

Ortega, A.; Rodríguez, D. \& Jiménez, A. (2013). Equilibrio trabajo-familia: corresponsabilidad familiar y autoeficacia parental en trabajadores de una empresa chilena. Diversitas: Perspectivas en Psicología, 9 (1), pp. 55-64.

Ortegón, E.; Pacheco, J. F. \& Prieto, A. (2005). Metodología del marco lógico para la planificación, el seguimiento y la evaluación de proyectos y programas. Santiago de Chile: Ilpes, Cepal.

Ortiz, L. G. (2009). La Convención de los Derechos del Niño veinte años después. Revista Latinoamericana de Ciencias Sociales, Niñez y Juventud, 7 (2), pp. 587619.

País, B. C. (2012). Las Ciencias Sociales ante la reeducación de menores: el programa Camino-Colonia y la participación ciudadana. Revista Educar para la Participación Ciudadana en la Enseñanza de las Ciencias Sociales. 2 (1), pp. 121-130. Recuperado de: http://didactica-cienciassociales.org/

País, B. C. \& González, X. M. S. (2014). Aprendiendo con menores en riesgo de exclusión. En J. P. i Blanch \&A. Santisteban (eds.) Una mirada al pasado y un proyecto de futuro: investigación e innovación en didáctica de las ciencias sociales, ( $\mathrm{pp}$. 355-362). Barcelona: AUPDCS, Universitat Autónoma Barcelona Servei de Publicacions. Recuperado de: http:// didactica-ciencias-sociales.org/

Peña, J. V.; Menéndez, C. R. \& Torío, S. (2010). Family and Socialization Processes: Parental Perception and Evaluation of 
Their Children's Household Labor. Journal of Comparative Family Studies, 41 (1), pp. 131-148.

República de Colombia (2006). Ley 1098 de Infancia y Adolescencia. Bogotá, D. C.: República de Colombia. Recuperado de: http://www.alcaldiabogota.gov.co/sisjur/ normas/Norma1.jsp? $\mathrm{i}=22106$

Sánchez, P. V. (2012). La experiencia vinculante afectiva del sujeto adolescente infractor. Revista Latinoamericana de Ciencias Sociales, Niñez y Juventud, 10 (1), pp. 453-465.

Serna, H. (2000). Gerencia estratégica: planeación y gestión-teoría y metodología. Bogotá, D. C.: 3R Editores.

Torres, L. C. \& Navarro, A. (2014). Técnicas prácticas de intervención psicosocial-Caja de herramientas. Bogotá, D. C.: Fundación Universitaria Luis Amigó. Recuperado de: http://virtual.funlam.edu.co/repositorio/ sites/default/files/CajadeHerramientas.pdf

Vilar, J. (2010). Reeducación social. La obra pedagógica de Josep Pedragosa. Educación Social: Revista de Intervención Socioeducativa, (44), pp. 139-141.

Villar, M. B. C.; Pernas, R. G. \& Iglesias, T. M. (2012). Servicios socioeducativos y corresponsabilidad en la conciliación de los tiempos familiares: situación de la infancia en la Galicia urbana. Pedagogía Social. Revista Interuniversitaria, (20), pp. 179-202. 